elevator the button of bone is detached, leaving the mucous lining of the sinus intact. This step is important for the following reasons. One or both sinuses may be healthy. The nature and character of the contents is obscured by admixture of blood. There may be no frontal sinus, and the cerebral cavity may be below what is supposed to be the lining membrane of the sinus. One or both sinuses, according to circumstances, is, or are, next to be opened with forceps and scissors and examined. Any secretion is to be removed. Any disease of the mucous membrane is to be treated by curetting or chloride of zinc, or both. Any polypi are to be removed, and the stumps treated with caustic, if requisite. Above all things, an opening must be established with the nasal cavity, and a drainage-tube inserted and maintained in position. Daily ablutions with antiseptic fluids will keep up a healthy action and assist a cure. The wound is to be accurately joined, the pericranium and pericranial aponeurosis, as well as the skin, being separately and carefully adjusted. The tube may protrude from the centre of the wound for the first few days for the purposes of irrigation, but subsequently may be shortened or dispensed with entirely. The result of this operation is usually immediately beneficial. The patients lose their troublesome symptoms and are permanently relieved. The wound bealing by first intention there is no scar or disfigurement, and the line of the incision is scarcely perceptible. The method adopted by ophthalmic surgeons of treating the other and more common class of cases as illustrated by a protrusion and displacement of the eyeball is rarely satisfactory. Lawson, Hulke, and others have laid down the rules and steps of the operation for opening the frontal sinuses at the upper and inner angle of the orbit at the most prominent point of the swelling with great exactitude. I have adopted this procedure seldom with more than temporary benefit, and never with speedy relief or cure. In this procedure the protrusion in the orbit is attacked and opened at its most prominent point. The incision is made through the orbicularis muscle, suspensory ligament of the eyelid, and often close to the pulley of the superior oblique muscle and in proximity to the lacrymal sac. The opening speedily closes and granulations spring up, impeding the discharges and ultimately bampering the movements of the lids and leaving a nasty scar and much disfigurement of the parts. More than this, during the operation no clear or exact view is obtained of the interior of the sinus or lining membrane. In this operation it is most difficult to find the infundibulum or enlarge the same, if required, from the orbital opening. $\Delta$ drainage-tube in the upper eyelid is most annoying to the patient and most unsightly. Experience shows that these cases treated thus go on discharging for years and are not cured. I have long discaroded opening the swelling in the orbit. I now always trephine in the front, a little to one side of the mid-line, and treat the case after the method of a latent empyema. Six weeks will usually suffice to cure these cases if operated on from the front. More rare affections of the frontral sinuses next to accumulation of flaids or pus are exostoses, polypi, and new growths. Exostoses are very rare and are usually of the hard description. There is one specimen in the Hunterian Museum, a large one, filling 'both sinuses and protruding into the nose and orbit. The museum at Cambridge contains another specimen, and a third is to be found at the museum of St. Bartholomew's Hospital. Mackenzie had two small specimens found, I believe, unexpectedly post mort $\epsilon \mathrm{m}$. Polypus of the frontal sinus of any size or in the form of pedunculated growths is rare, but when occurring is usually associated with the same disease in the nose. Mr. Hulke ${ }^{1}$ reported several cases of polypus of the frontal sinus, only one case of which was unassociated with a similar state of things in the nose. These cases generally led to accumulation in the sinuses and were not primarily diagnosed as polypi. New growths are extremely rare, but sarcoma has been repcrted as presumably originating in these spaces. Of insects in the frontal sinus cases have been reported by Mackenzie and others. One interesting case of foreign body in the frontal sinus was under my own care-the only case of the kind, so far as I am aware, on record. During the exhibition of snuff a quantity was drawn into an unusually patent frontal sinus, and set up the most intense inflammation with great headacbe, high temperature, and delirium.

Harley-street, W.

I The LaNCBT, March 14th, 1891.

\section{OBSERVATIONS UPON RE-VACCINATION.}

\section{BY ROBERT J. CARTER, M.D. LOND., D.P.H. ENG.}

DURING a recent small. pox epidemic I collected notes of 319 cases of re-vaccination. Calf lymph prepared with glycerine was used in every case, and the operation was performed indiscriminately on all persons who presented themselves, the ages varying from four to sixty years. Four insertions were made in the usual situation on the arm in every case, and only those arms which presented a vesicular stage on the eighth day after the operation were accounted successful. At the same time short notes as to the clinical condition of the patients were taken. The results obtained show very clearly how rapidly in early life the effects of primary vaccination wear out, the insusceptibility to revaccination being of a much more evanescent character than is generally believed. For the purpose of showing this clearly I have arranged the successtul cases in two Tables. Table A comprises 148 children up to, and iccluding fourteen years of age, all vaccinated in infancy; Table B comprises 171 adults over twenty-four years of age, vaccinated later in life than the fourteenth year.

TABLE A.

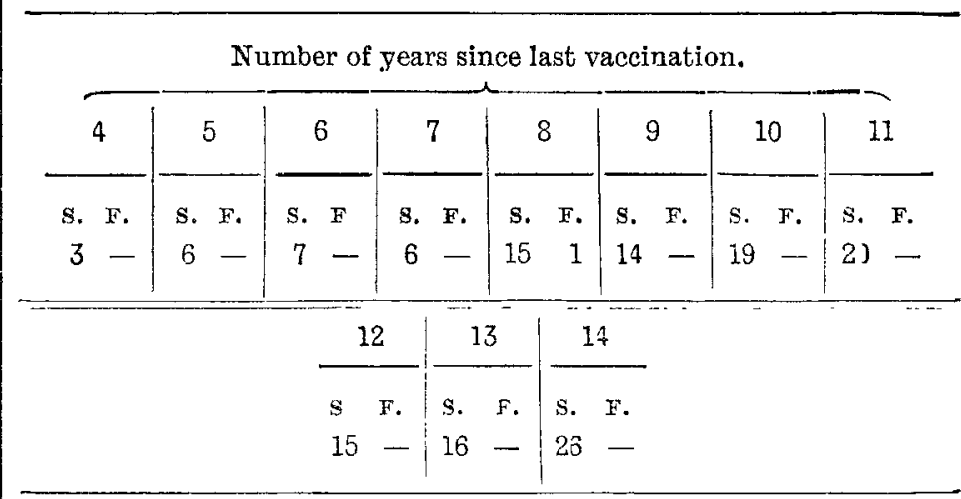

TABLE B.

\begin{tabular}{|c|c|c|c|c|c|c|}
\hline \multicolumn{7}{|c|}{ Number of years since last vaccination. } \\
\hline 1 & 2 & 3 & 4 & 5 & 6 & 7 \\
\hline s. $\quad F$. & s. F. & s. F. & s. F. & S. F. & $\therefore \quad F$. & S. F. \\
\hline-12 & -13 & -6 & -8 & -2 & -6 & -1 \\
\hline 8 & 9 & 10 & 11 & 12 & 13 & 14 \\
\hline S. F. & s. F. & B. F. & S. F. & S. F. & S. F. & s. F. \\
\hline 14 & 12 & 22 & $1-$ & 12 & 28 & -3 \\
\hline 15 & 16 & 17 & 18 & 19 & 20 & Over 20 \\
\hline s. $F$. & s. F. & S. F. & S. F. & S. F. & s. F. & S. $\mathrm{r}$. \\
\hline 21 & -2 & -- & $1-$ & -2 & -- & 824 \\
\hline
\end{tabular}

Comparing these resulta somewhat in detail, it will be noted that of those under fourteen years of age vaccinated in infancy the earliest successful case is four years after the primary vaccination, whilst in Table $B$ the earliest successful vaccination is after a lapse of eight years. In Table A the only three cases vaccinated at the fourth year were all successful ; in Table B the earliest successful case occurs in the eighth year after previous vaccination. The proportion of failures to successes gradually diminishes in Table B. In Table A the solitary unsuccessful case is probably an accident. Practically at the tenth year revaccination of children vaccinated in infancy always results in a more or less typical vaccinia, whereas in adults it is not until about twenty years after a previous vaccinationand not always then-we can ensure any successful result. I feel that the paucity of numbers and possibilities of failures due to the vaccinator tend to depreciate these results, but when allowance has been made for such deficiencies I consider they still will bear the interpretation I have put upon them-viz, the greater rapidity with which the effects of vaccinia upon the organism wear out 
in early life compared to more mature age. The explanation of this, I venture to suggest, is that up to puberty the tissues are in a much more active condition; tissue change is more rapid, and impressions on the organism more easily effaced than in adult life.

The Local Government Board do not allow public vaccinators to be paid for re-vaccinations upon children under ten years of age; but in the face of these results I would suggest that in small-pox epidemics every child in whose case four years have elapsed since the primary vaccination should be re-vaccinated. The condition of the arm on the eighth day after re-vaccination varies exceedingly. The areola appears much earlier than in primary vaccination. In 197 cases it was present on the second day. On the eighth day it varies from a circumferential blush around the vesicles to a uniform redness extending from the shoulder to the elbow, of a bright crimson colour, disappearing on pressure. I regard this condition as different to that in which we see odema and redness extending down to the finger-tips. Some enlargement of the axillary glands can always be discovered if looked for. In 106 cases these glands were large, hard and tender, and in some instances exquisitely painful. In three cases the glands above the clavicle were also affected. Lymphangitis was present in nine cases, the lymphatic vessels being felt as hard, swollen, tender cords along the course of the axillary vessels. Induration and cedema of the arm were present in ninety-eight cases, and were of a curiously persistent character. They did not appear to be associated entirely with the glandular affection, but were apparently dependent upon the intensity of the local inflammation at the site of the vaccination.

Constitutional symptoms, except the temperature, are difficult to estimate, apart from the idiosyncrasy of the patient. Several complained of aching pain in the lumbar region, headache and nausea which, while not uncommon symptoms of a febrile condition, are the cardinal symptoms of the early stage of variola. Lassitude, giddiness, and loss of appetite were common among the male adults, whilst fainting and neurotic manifestations were noticed among the females. Post-vaccinal rashes were only seen in four instances. It was impossible to take the temperature of all the cases, but in those in which fever was noted the pyrexia appeared directly proportional to the amount of inflammatory action in the arm. It is commonly accepted that the amount of inflammatory action depends to some extent on the active use of the arm during the week following vaccination. In all my cases the occupation was noted, and it was found that the most severe cases occurred in women who had worn slings directly the vaccination showed signs of being successful. Its severity appears to me to depend entirely upon the general constitutional condition and the environment of the individual.

The treatment of severe cases was simple and apparently successful-free saline purgation, rest for the arm, the application of a lead and opium lotion, early and frequent removal of the scabs, and a boracic ointment dressing for the subjacent ulcers.

Acacia-road, N.W.

THE CONTINUOUS ADMINISTRATION OF NITROUS OXIDE GAS AND OXYGEN DURING MINOR SURGICAL OPERATIONS.

BY H. BELLAMY GARDNER, M.R.C.S. ENG, L.R.C.P. LOND.,

ASSISTANT ANASTEETIST TO CHARING-CROSS HOSPITAE,

ErGHT years have elapsed since the possibility and distinct advantage of giving small percentages of oxygen with nitrous oxide gas to produce anæsthesia at ordinary atmospheric pressures were first pointed out in THE LANCET by Dr. Frederic Hewitt. ${ }^{1}$ Though this method has now obtained a very wide and substantial footing in the dental profession, it can hardly be said that its remarkable value in prolonging the anæsthesia and rendering the patient flaceid and free from spasm for several minutes at a time has procured sufficient recognition among surgeons, who woulc have been expected to readily appreciate a mixture which leaves neither nausea nor mental distarbance after its inbalation, and, more important still, which subjects a patient to the least possible risk incurred by the exhibition of any known anæsthetic agent. The presence and concurrent inhalation of from 10 to 15 per cent. of oxygen with nitrous oxide gas are sufficient to maintain the due proportion of oxy-hæmoglobin in the arterial blood, and thereby to render an inhalation which formerly was cut short by the supervention of asphyxial symptoms capable of being sustained while many minor surgical operations are being effected. While the condition produced by the presence of oxygen is clinically free from asphyxial symptoms in those surgical procedures which are undertaken after removal of the face-piece, I have also found that a very tranquil anæsthesia may be obtained in those others which permit of its continuous application, even with quantities of oxygen rather larger than those usually administered in the first class of cases. To illustrate the kind of painful operation for which I think this method has proved to be appropriate the following cases are taken from my notebooks.

CASE 1.-A man, aged forty-seven years, with good chest expansion, stoutly built, and of rather florid colour, required the removal of four sebaceous cysts from the scalp. Before the administration he was seated in a comfortable armchair. The surgeon washed and prepared the skin around the cysts, and the face-piece was then applied. Commencing with a small percentage of oxygen (to allow for that already present in the lungs), the amount was gradually increased whilst the lid-reflex disappeared. Breathing continued deep and free as during sleep; the colour was good; no inconvenient movement occurred; and the operation-during which the cysts were excised and the incisions sewn up-proceeded for five and a half minutes. At its completion the patient awoke-one minute after removal of the face-piece-without any untoward sensations whatever.

CASE 2.-A girl, aged seventeen years, of average height and build but rather anæmic, suffering from a tuberculous abscess in the left side of the neck, was seated in an upright chair with a head-rest while the swelling was incised, thorougbly scraped, cleaned out, and then sutured with four separate stitches. During this operation, which lasted four and a half minutes, she was kept absolutely tranquil with a good, regular pulse under the influence of nitrous oxide gas and oxygen. The recovery was rapid and presented no after-effects of any kind.

CASE 3.-A man, aged thirty-five years, of average height but rather thin and pale, had two suppurating cysts on the scalp, one of which was very painful. He was seated in an easy-chair when the face-piece was applied. Again the percentages of oxygen with the gas were gradually increased until he was softly snoring as if under chloroform. The pupil remained of moderate size and the pulse and colour were very good whilst the incision, scraping, and dressing of the inflamed areas were completed. This operation also lasted four and a half minutes. He afterwards said it was very pleasant to inhale, that he felt quite well, and had not the slightest sensation or consciousness during the administration.

In the course of my work at Charing-cross Hospital I have lately administered nitrous oxide gas and oxygen during the excision of a varicocele lasting fourteen minutes. The patient, who was a male, aged twenty-two years, of a rather florid type, was inclined now and then to become a trifle dusky in colour, but this was owing to there not being quite sufficient oxygen at command; his absolute flaccidity and quietude during the time were, however, quite satisfactory to the operator. His breathing remained regular and the circulation was well maintained throughout. Recovery was complete in two minutes and he was not sick subsequently.

It has been my custom for the past two years to administer this ansesthetic in the aural department for the removal of post-nasal adenoid growths, and the advantages which may be claimed for its use are: (1) that it is not attended with danger to life; (2) that preparation for the operation is not absolutely necessary, though undoubtedly the best results are obtained after abstinence from food for three or four hours (3) that hrmorrhage is unaffected by it ; (4) that the jactitation and cyanosis produced by pure nitrous oxide gas are absent; (5) that any posture desired by the operator may be safely astumed; (6) that when the face-piece is remcred the available anæsthesia is about fifteen seconds longer than that 